\title{
A Role for Peroxisome Proliferator-Activated Receptors in the Immunopathology of Schistosomiasis?
}

\author{
Barrie J. Anthony, ${ }^{1}$ Jeremy T. Allen, ${ }^{2}$ Yuesheng S. Li, ${ }^{1}$ and Donald P. McManus ${ }^{1}$ \\ ${ }^{1}$ Queensland Institute of Medical Research, Post Office Royal Brisbane Hospital, QLD 4029, Australia \\ ${ }^{2}$ Department of Biology, Centre for Parasitology and Disease, Biomedical Sciences Research Institute, University of Salford, \\ Manchester M5 4WT, UK
}

Correspondence should be addressed to Barrie J. Anthony, barrie.anthony@qimr.edu.au

Received 1 December 2010; Accepted 27 February 2011

Academic Editor: Marion M. Chan

Copyright (C) 2012 Barrie J. Anthony et al. This is an open access article distributed under the Creative Commons Attribution License, which permits unrestricted use, distribution, and reproduction in any medium, provided the original work is properly cited.

Peroxisome proliferator-activated receptors (PPARs) have been demonstrated to have a role in immune regulation. In general, they are anti-inflammatory and promote Th2 type responses, and they are associated with the alternative activation of macrophages. Interestingly, helminth infections, such as the schistosome blood flukes that cause schistosomiasis, are characterised by a Th2 response and the accumulation of alternative activated macrophages. This would suggest that at some level, PPARs could have a role in the modulation of the immune response in schistosomiasis. This paper discusses possible areas where PPARs could have a role in this disease.

\section{Introduction}

The peroxisome proliferator-activated receptors (PPARs) are a group within the 48 transcription factors of the nuclear hormone receptor family involved in lipid metabolism and inflammation [1]. To be transcriptionally active, they require hetrodimerisation with the retinoid X receptor (RXR) to which the resulting heterodimers bind with peroxisome proliferator-response elements (PPREs) on DNA after activation by a ligand-to-modulate transcription [2]. The PPREs are located at the $5^{\prime}$ end of the target gene and consist of a repeat sequence-AGGTCA - separated by one nucleotide [3]. The binding to the PPRE is orientated with PPAR at the $5^{\prime}$ end and RXR towards the $3^{\prime}$ end [2]. For transcriptional control to occur, the PPAR/RXR heterodimers have to interact with coactivators or suppressors for stimulation or inhibition of target-gene expression, respectively [2]. The PPARs can also block transcription of other genes by interacting with other transcription factors by nongenomic transrepression, whereby they inhibit transcription by preventing dissociation of corepressors or sequester coactivators needed for binding of the transcription factor to the DNA [4]. There are 3 isoforms of the PPAR receptors, $\operatorname{PPAR} \alpha, \operatorname{PPAR} \beta / \delta$, and PPAR $\gamma$ [5]. PPAR $\alpha$ is expressed in the liver, brown fat, heart, and skeletal muscle which have high levels of fatty acid catabolism, while PPAR $y$ is expressed in adipose tissue, the colon, and in macrophages, it is the major regulator in adipocyte differentiation and is a determinant in insulin sensitivity [3]. PPAR $\beta / \delta$ is ubiquitously expressed and is thought to have a role in metabolic disorders [3]. Polyunsaturated fatty acids and eicosanoids act as natural ligands for these receptors; however, synthetic ligands exist such as fibrates that target PPAR $\alpha$ and the thiazolidinediones that target PPAR $\gamma$ [3].

PPARS have been demonstrated to be important in a number of different disease states such as metabolic disorders [3], inflammation [6], malaria [7], Chagas disease [8], and leishmaniasis [9]. Recent studies have revealed a role for PPARs in the control of the immune response. In general, they are anti-inflammatory [10], promote the development of alternatively activated macrophages $(\mathrm{AAM} \Phi)$ [11], and are Th2 biasing [12]. Helminth worms have an incredible ability to modulate the host immune response and, in general, promote a Th2-biased environment that commonly involves 
the generation of AAMФs [13], as occurs during schistosome infection. The fact that this parasite and other helminths induce Th2 biasing, with accumulation of $A A M \Phi s$, suggests that at some level, PPARs could be involved. This paper will explore the current state of knowledge in this area, focusing on the role of PPARs in the immunopathology of schistosomiasis and their potential as novel therapeutic targets.

\section{Immune Regulation in Schistosomiasis}

Schistosomiasis is a major health problem responsible for significant morbidity and mortality worldwide. It is estimated that approximately 200 million people are infected, causing severe disease in 20 million people [14]. The disease is caused by infection with the trematode worms, the schistosomes, of which Schistosoma mansoni, S. japonicum, and $S$. haematobium are the most important in regards to human disease [15]. Pathology is associated with the host's immune response to the eggs which results in a granulomatous reaction [16]. Helminth parasites are able to modulate the host immune response, allowing them to have good longevity within the mammalian host. Helminth infections are noted for polarising the immune response more towards a Th2 response characterised by interleukin (IL)-4, 5, and 13, large amounts of IgE, and by CD4 ${ }^{+}$ $\mathrm{T}$ cells [17]. In schistosomiasis, the immune response is characterised by a switch from an early proinflammatory Th1 response to a Th2 response to eggs released by the female worm [18]. The Th2 response has the characteristics described above and is associated with $\mathrm{AAM \Phi s} \mathrm{([19-21]).}$ Cytokines such as INF- $\gamma$, IL-2, IL-12, and TNF- $\alpha$ are associated with the early Th1 response and are repressed during this switch [18]. Helminth parasites can achieve this modulation the by release of soluble factors which can interact with host immune cells [13]. There is good evidence for this in schistosome infections in which both live or dead eggs injected into naive mice rapidly induce a Th2 response [20]. Egg-derived products have been observed to drive the switch from the Th1 response to the Th2 response. Examples of this are the IL-4-inducing principal of $S$. mansoni eggs (IPSEs) that is secreted from the egg subshell into the surrounding granuloma area and has been demonstrated to induce human basophils to produce IL-4 and IL-13 [22]. Similarly, the glycoprotein, omega-1 that is secreted by S. mansoni eggs and present in secreted egg antigen (SEA), has been observed to drive human monocyte-derived dendritic cells towards Th2 polarisation and to generate Th2 responses in vivo in mice [23]. Egg-derived glycoconjugates, $\alpha 3$-fucosyltransferase, and core g 2-xylosyltransferase have been used with dendritic cells to produce a Th2 response in the murine model of disease caused by $S$. mansoni [24].

\section{Immune Regulation by PPARs}

It is believed that PPARs may be important in the regulation of the immune response, a role supported by the fact that
PPARs have been described in monocytes, macrophages, neutrophils, peripheral blood lymphocytes, T cells, B cells, natural killer cells, dendritic cells, eosinophils, and mast cells [1]. Supporting this, ligands of PPARs have been shown to have a therapeutic role in several models of inflammatory and autoimmune diseases [6]. PPAR $y$ agonists have been demonstrated to have anti-inflammatory effects in renal injury [25], murine carotid atherosclerosis [26], and in oxidative stress induced in a human diploid fibroblast model of aging [27]. PPAR $\beta / \delta$ agonists have additionally been demonstrated to have a protective role in a murine model of autoimmune encephalomyelitis [28]. Further, PPAR $\gamma$ agonists have been observed to inhibit the production of TNF- $\alpha$ in human monocytes [10].

Part of the anti-inflammatory mode of action of PPARs is due to the fact they can interact with transcription factors involved in inflammation such as NF- $\kappa \mathrm{B}$, activator protein-1 (AP-1), and signal transducers and activators of transcription (STAT) at a transcriptional level $([29,30])$. In the case of NF- $\kappa$ B and AP-1, PPAR $\alpha$ has been shown to interact directly with p65, c-Jun, and CBP, thereby interfering with their transcriptional capacity [29], while the PPAR $\gamma$ agonist, 15dPGJ2, inhibits STAT signalling indirectly [30]. Interference of these pathways results in the downregulation of the Th1 proinflammatory cytokines TNF- $\alpha$, IL-1, -6 , and 12 ([29, 30]).

Notably, PPARs have been demonstrated to result in upregulation of Th-2 responses and downregulation of Th1 responses. An agonist of $\operatorname{PPAR} \alpha$, gemfibrozil, results in increased number of GATA3 positive T cells in the spleens of donor mice as well as the stimulation of its expression and DNA-binding activity resulting in IL-4 production [12]. In the same study, gemfibrozil was additionally observed to inhibit the expression and DNA-binding activity of Tbet, causing a decrease in INF- $\gamma$ production. IL-4 can interact with PPAR $\gamma$ indirectly and directly in macrophages [31]. PPAR $\gamma$ expression is both directly and indirectly upregulated by IL- 4 . IL- 4 will induce target-gene expression by increasing PPAR $y$ expression and by increasing the production of PPARy ligands via 15-lipoxygenase, which results in lipoperoxidation products such as linoleic acid (HODE) or arachidonic acid (HETE) [31]. Schistosomes and other helminths could potentially interact with PPARs through these pathways. They could do this indirectly via IL-4 and IL-13 as both cytokines can activate PPAR $y$ resulting in the suppression of the proinflammatory response and activation of $A A M \Phi$ s which favour the establishment of a chronic parasite infection [1]. Schistosomes could potentially interact with PPARs via hemozoin. Schistosomes produce hemozoin as a product from feeding on mammalian host red blood cells; its structure is identical to malarial hemozoin [1]. It is composed of a complex mixture of neutral lipids and polyunsaturated lipids from which lipoperoxidation products HETE and HODE acid, which are natural ligands for PPAR [1], are derived (Figure 1). Carter et al. [32] showed that macrophages that have previously phagocytosed schistosomal-derived hemozoin have a reduced ability to produce iNOS in response to LPS. 


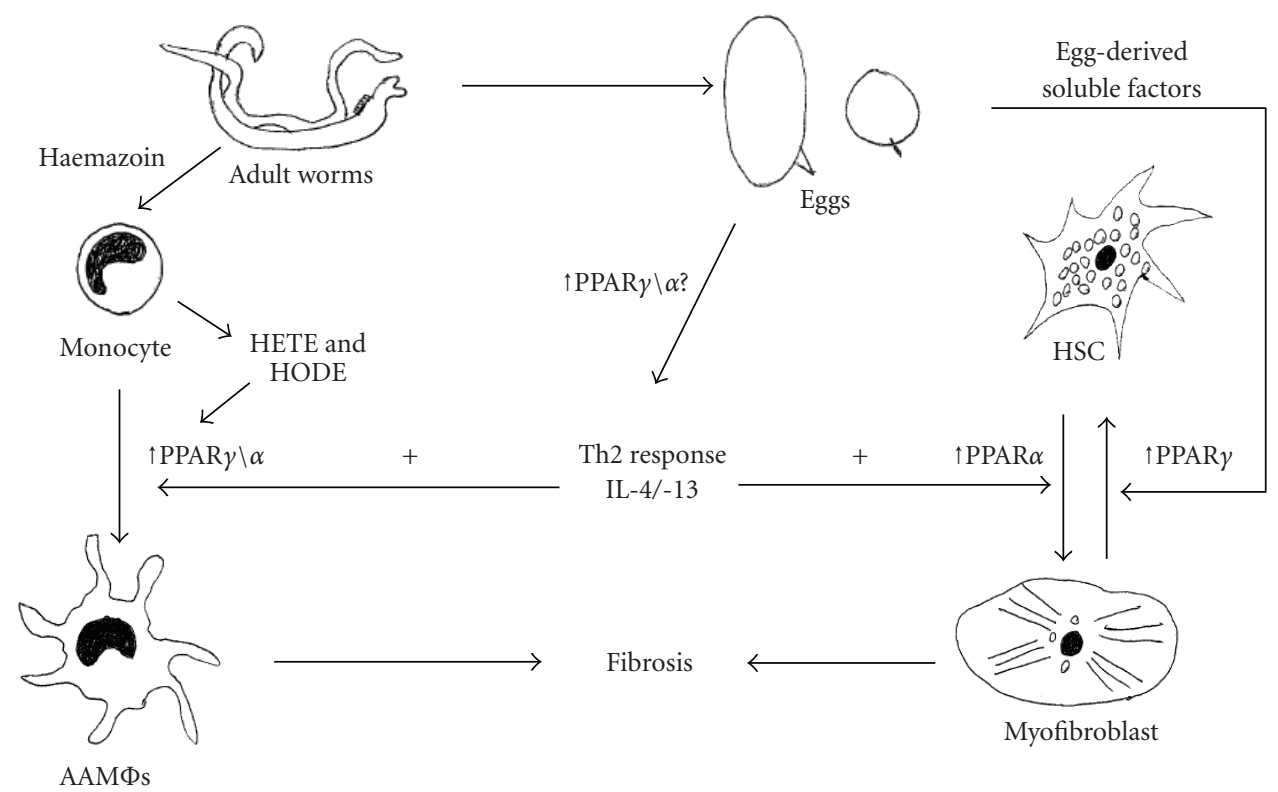

FIGURE 1: Summary of possible interactions in schistosomiasis with PPARs. This figure displays the possible pathways in which PPARs could be involved in schistosomiasis. PPARs could be involved in immune regulation, as they are associated in generation of a Th2 response. PPAR $\beta \gamma / \alpha$ both cause downregulation of Th1 cytokines and promote IL-4/-13 production. PPARs have a role in the alternate activation of macrophages where PPAR $\gamma / \alpha$ have been demonstrated to be essential for this process. In schistosomiasis, AAMФs have a protective effect and a role in Th2 biasing. Schistosomes could interact with this activation indirectly via induction of IL-4/-13 production and directly by the breakdown products of hemozoin, which can interact with PPAR $\gamma / \alpha$. In terms of pathology, the PPARs could interact with the transdifferentiation process of HSCs into fibrogenic myofibroblasts. They could limit this process by inhibiting transdifferentiation associated with increased PPAR $\gamma$, whereas PPAR $\alpha$ would be associated with generation of the fibrogenic myofibroblast.

\section{PPARs and Alternatively Activated Macrophages}

Macrophages have multiple roles with regards to the host immune response. They have a role in early detection of invading pathogens, both as antigen presenting cells (APCs) that initiate a host response and as effecter cells that can act to kill the invading pathogen [33]. Macrophages used to be classified as either activated or deactivated, but in recent years, this has changed to classically activated macrophages (CAMФ) and AAMФs [33]. CAMФs are induced by INF$\gamma$, TNF- $\alpha$, and LPS and produce proinflammatory cytokines such as IL-1 $\beta$, IL-12, IL-23, and TNF- $\alpha$ and the chemokines CXCL-9, -10,-11, and -16 [33]. AAMФs are associated with production of IL-10 and are induced by IL- 4 and IL13 [33]. This classification has been expanded in recent years with proinflammatory macrophages being termed M1, while anti-inflammatory macrophages are termed M2. The M2 macrophages have been divided into different subsets, whereby AAMФs are classified as M2a cells which are defined by low expression levels of IL-12 [34], or are M2b cells, which release high levels of IL-10 on activation by immune complexes, and M2c cells which are induced by IL-10 and are believed to be more similar to САМФ [33]. One of the main differences distinguishing $А А М Ф s$ from CAMФs is in how they metabolise L-arginine. CAM $\Phi$ metabolise L-arginine into NO via iNOS, while AAMФ metabolise L-arginine into urea and L-ornithine via arginase-1 (arg-1) [35].
AAM $\Phi$ have been associated with many helminth infections with many different roles attributed to them. In infection with the nematode Brugia malayi, AAMФs are associated with Th-2 biasing [36, 37], while in infection with Heligmosomoides polygyrus, AAM $\Phi$ s have a role in parasite clearance and host protection [38]. In cestode infections, AAM $\Phi$ s have been associated with downregulation of the immune response in Echinococcus multilocularis [39] and Th2 biasing in Taenia crassiceps infection as well as favouring parasite survival [40]. In Schistosoma infection, they have a role in Th-2 biasing as well as in downregulation of the Th-1 response and mediate immunopathology promoting host protection, but, at the same time, they promote progressive pathology due to granuloma formation ([21, 41]). Overall, their role seems to be host protective by causing downregulation of overaggressive inflammatory reactions, but they are also protective for the parasite, forming part of the immunomodulation strategy needed for successful colonisation of the host.

PPARs could potentially have a role in helminth infections by regulating AAMФs. There are a number of studies demonstrating PPAR $\gamma$ as essential for AAM $\Phi$ activation and maturation in other disease states such as metabolic syndrome and leishmaniasis $([9,11])$. In metabolic syndrome, it has been shown with macrophage-specific PPAR- $\gamma$ knockout mice that PPAR $\gamma$ is essential for AAM $\Phi$ maturation resulting in the mice developing diet-induced obesity, insulin resistance, and glucose intolerance [11]. Additionally, PPAR $\gamma$ and 
PPAR $\delta$ agonists have been observed to mediate arginase-1 expression in macrophages, and this expression is blocked in macrophages from PPAR $\gamma$ - and $\operatorname{PPAR} \delta$-deficient mice [11]. Interactions between IL-4/13 and PPARs have been extensively studied in leishmaniasis in which PPARs promote AAMФ-mediated susceptibility to the disease by stimulating intracellular amastigote growth in infected macrophages [11]. This is due to the lack of NO production in resultant $\mathrm{AAM} \Phi$ which is essential for amastigote killing.

\section{DO PPARs Modulate Host Pathology?}

The hepatic stellate cell (HSC) is located within the liver sinusoid in the space of Disse where it is responsible for storage of vitamin $\mathrm{A}$ and the maintenance of a lowdensity matrix between the liver endothelium cells and the hepatocytes [42]. Maintenance of this matrix is important as it allows solutes in the plasma to reach the hepatocytes unimpeded, allowing the liver to function correctly [43]. In response to insult or injury to the liver, HSCs can undergo a process of transdifferentiation from the quiescent vitamin A-storing cell to a myofibroblast responsible for the accumulation of scar tissue within the space of Disse [44]. This has highlighted parallels between this cell type and that of the adipocyte which can undergo a similar process [45]. Adipocytes differentiate from a fibroblast-like preadipocyte and become lipid laden associated with the expression of PPAR $\gamma$ [46]. Quiescent HSCs express PPAR $\gamma$ which upon transdifferentiation into a myofibroblast-like cell lose their ability to store lipid droplets as the expression and activity of PPAR $\gamma$ decrease [47]. This has suggested a role for PPAR $\gamma$ agonists in the treatment of fibrosis. PPAR $\gamma$ agonists have been demonstrated to cause reversion of the myofibroblast back into a quiescent HSC $([45,47])$.

Recent studies have implicated a role for HSCs in the pathogenesis of schistosomiasis [48]. Activated HSCs have been observed in the murine model of disease and at the end stage of human disease with S. japonicum [49] and human disease with S. mansoni [50]. Notably, the PPAR $y$ agonist rosiglitazone has been demonstrated to prevent fibrosis in S. japonicum infection of mice [51]. In this study, mice cotreated with the antischistosome drug praziquantel and rosiglitazone induced reduced expression of collagen 1 and 3, $\alpha$ smooth muscle actin (a marker for myofibroblasts), inflammation, increased expression of PPAR $\gamma$, reduced NF$\kappa \mathrm{B}$-binding activity, and reduced TNF- $\alpha$ levels [51]. In a recent study, Anthony et al. [52] showed that eggs of $S$. mansoni could downregulate fibrogenesis in the human HSC cell line, LX-2, causing regression from the activated myofibroblast to the quiescent HSC. This downmodulation was associated with increased expression of PPAR $y$ at the gene level as well as with the accumulation of lipid droplets within the cytoplasm of HSCs. At the granuloma level, fibrosis first occurs towards the periphery of the granuloma site, and it was postulated in this study that antigens from the egg may inhibit fibrosis in close proximity to the egg as it is not until the egg is killed and destroyed that fibrosis occurs throughout the granuloma area. However, PPAR $\gamma$ could act as a double-edged sword, as it would be involved in alternative activation of macrophages at the granuloma site, which in turn can be responsible for collagen production by the production of arginase- 1 which promotes the production of proline. Additionally, the Th2 response is profibrogenic and high levels of IL-13 are associated with fibrosis in schistosomiasis [53].

\section{Conclusions}

Schistosomiasis is characterised by a switch from an early Th1 response to a Th2 response and accumulation of AAMФs in response to eggs released by the schistosome worms. PPARs have been demonstrated to cause downregulation of proinflammatory Th1 cytokines while simultaneously upregulating Th2 responses. They have additionally been shown to be essential in the alternative activation of macrophages. This suggests that PPARs may play a role in the regulation of the host response to schistosome antigens. Additionally, it has been demonstrated that S. mansoni eggs cause downregulation of fibrogenesis in the humanderived HSC cell line, LX2, a response associated with increased expression of PPAR $\gamma$, and accumulation of lipid droplets within the cell's cytoplasm. Rosiglitazone, a PPAR $\gamma$ ligand, has been additionally been demonstrated to reduce pathology associated with S. japonicum infection in mice. The possible interactions with schistosomiasis and PPARs are summarised in Figure 1. Further studies of the role of PPARs in this disease and those caused by other helminth infections are, therefore, warranted and may help in the identification of new antipathology drug and vaccine targets for schistosomiasis and other important diseases caused by the parasitic helminths.

\section{Conflict of Interests}

The authors declare that they have no Conflict of interests.

\section{Acknowledgments}

Research on the pathogenesis of schistosomiasis by Y. S. Li and D. P. McManus is funded by the National Health and Medical Research Council (NHMRC) of Australia and the DANA Foundation, USA. Y. S. Li is an Australian Research Council Future Fellow and a Howard Hughes (USA) Medical Institute International Research Scholar. DM is a Senior Principal Research Fellow of the NHMRC (Australia).

\section{References}

[1] M. M. Chan, K. W. Evans, A. R. Moore, and D. Fong, "Peroxisome proliferator-activated receptor (PPAR): balance for survival in parasitic infections," Journal of Biomedicine \& Biotechnology, vol. 2010, Article ID 828951, 2010.

[2] L. Michalik, J. Auwerx, J. P. Berger et al., "International union of pharmacology. LXI. Peroxisome proliferator-activated receptors," Pharmacological Reviews, vol. 58, no. 4, pp. 726$741,2006$. 
[3] M. Vázquez-Carrera, T. Coll, E. Barroso et al., "The role of peroxisome proliferator-activated receptor $\beta / \delta$ on the inflammatory basis of metabolic disease," PPAR Research, vol. 2010, Article ID 368467, 2010.

[4] N. S. Tan, L. Michalik, B. Desvergne, and W. Wahli, "Multiple expression control mechanisms of peroxisome proliferatoractivated receptors and their target genes," Journal of Steroid Biochemistry and Molecular Biology, vol. 93, no. 2-5, pp. 99105, 2005.

[5] G. Rizzo and S. Fiorucci, "PPARs and other nuclear receptors in inflammation," Current Opinion in Pharmacology, vol. 6, no. 4, pp. 421-427, 2006.

[6] A. Yessoufou and W. Wahli, "Multifaceted roles of peroxisome proliferator-activated receptors (PPARs) at the cellular and whole organism levels," Swiss Medical Weekly, vol. 140, p. w13071, 2010.

[7] L. Serghides and K. C. Kain, "Peroxisome proliferatoractivated receptor $\gamma$-retinoid $\mathrm{X}$ receptor agonists increase CD36-dependent phagocytosis of Plasmodium falciparumparasitized erythrocytes and decrease malaria-induced TNF$\alpha$ secretion by monocytes/macrophages," Journal of Immunology, vol. 166, no. 11, pp. 6742-6748, 2001.

[8] F. Nagajyothi, M. S. Desruisseaux, L. M. Weiss et al., "Chagas disease, adipose tissue and the metabolic syndrome," Memorias do Instituto Oswaldo Cruz, vol. 104, no. 1, pp. 219-225, 2009.

[9] A. Gallardo-Soler, C. Gómez-Nieto, M. L. Campo et al., "Arginase I induction by modified lipoproteins in macrophages: a peroxisome proliferator-activated receptor$\gamma / \delta$-mediated effect that links lipid metabolism and immunity," Molecular Endocrinology, vol. 22, no. 6, pp. 1394-1402, 2008.

[10] C. Jiang, A. T. Ting, and B. Seed, "PPAR- $\gamma$ agonists inhibit production of monocyte inflammatory cytokines," Nature, vol. 391, no. 6662, pp. 82-86, 1998.

[11] J. I. Odegaard, R. R. Ricardo-Gonzalez, M. H. Goforth et al., "Macrophage-specific PPAR $\gamma$ controls alternative activation and improves insulin resistance," Nature, vol. 447, no. 7148, pp. 1116-1120, 2007.

[12] S. Dasgupta, A. Roy, M. Jana, D. M. Hartley, and K. Pahan, "Gemfibrozil ameliorates relapsing-remitting experimental autoimmune encephalomyelitis independent of peroxisome proliferator-activated receptor- $\alpha$," Molecular Pharmacology, vol. 72, no. 4, pp. 934-946, 2007.

[13] J. P. Hewitson, J. R. Grainger, and R. M. Maizels, "Helminth immunoregulation: the role of parasite secreted proteins in modulating host immunity," Molecular and Biochemical Parasitology, vol. 167, no. 1, pp. 1-11, 2009.

[14] L. Chitsulo, D. Engels, A. Montresor, and L. Savioli, "The global status of schistosomiasis and its control," Acta Tropica, vol. 77, no. 1, pp. 41-51, 2000.

[15] A. G. P. Ross, P. B. Bartley, A. C. Sleigh et al., "Schistosomiasis," New England Journal of Medicine, vol. 346, no. 16, pp. 12121220, 2002.

[16] M. L. Burke, M. K. Jones, G. N. Gobert, Y. S. Li, M. K. Ellis, and D. P. McManus, "Immunopathogenesis of human schistosomiasis," Parasite Immunology, vol. 31, no. 4, pp. 163$176,2009$.

[17] P. G. Thomas and D. A. Harn Jr., "Immune biasing by helminth glycans," Cellular Microbiology, vol. 6, no. 1, pp. 1322, 2004.

[18] E. J. Pearce and A. S. MacDonald, "The immunobiology of schistosomiasis," Nature Reviews Immunology, vol. 2, no. 7, pp. 499-511, 2002.
[19] A. R. de Jesus, A. Magalhães, D. Gonzalez Miranda et al., "Association of type 2 cytokines with hepatic fibrosis in human Schistosoma mansoni infection," Infection and Immunity, vol. 72, no. 6, pp. 3391-3397, 2004.

[20] E. J. Pearce, "Priming of the immune response by schistosome eggs," Parasite Immunology, vol. 27, no. 7-8, pp. 265-270, 2005.

[21] D. R. Herbert, C. Hölscher, M. Mohrs et al., "Alternative macrophage activation is essential for survival during schistosomiasis and downmodulates $\mathrm{T}$ helper 1 responses and immunopathology," Immunity, vol. 20, no. 5, pp. 623-635, 2004.

[22] G. Schramm, F. H. Falcone, A. Gronow et al., "Molecular characterization of an interleukin-4-inducing factor from Schistosoma mansoni eggs," Journal of Biological Chemistry, vol. 278, no. 20, pp. 18384-18392, 2003.

[23] B. Everts, G. Perona-Wright, H. H. Smits et al., "Omega-1, a glycoprotein secreted by Schistosoma mansoni eggs, drives Th2 responses," Journal of Experimental Medicine, vol. 206, no. 8, pp. 1673-1680, 2009.

[24] C. Faveeuw, T. Mallevaey, K. Paschinger et al., "Schistosome Nglycans containing core $\alpha 3$-fucose and core $\beta 2$-xylose epitopes are strong inducers of Th2 responses in mice," European Journal of Immunology, vol. 33, no. 5, pp. 1271-1281, 2003.

[25] W. Arozal, K. Watanabe, P. T. Veeraveedu et al., "Telmisartan prevents the progression of renal injury in daunorubicin rats with the alteration of angiotensin II and endothelin1 receptor expression associated with its PPAR- $\gamma$ agonist actions," Toxicology, vol. 279, no. 1-3, pp. 91-99, 2011.

[26] K. Chang, S. A. Francis, E. Aikawa et al., "Pioglitazone suppresses inflammation in vivo in murine carotid atherosclerosis: novel detection by dual-target fluorescence molecular imaging," Arteriosclerosis, Thrombosis, and Vascular Biology, vol. 30, no. 10, pp. 1933-1939, 2010.

[27] Y.-H. Lee, N.-H. Lee, G. Bhattarai et al., "PPAR $\gamma$ inhibits inflammatory reaction in oxidative stress induced human diploid fibloblast," Cell Biochemistry and Function, vol. 28, no. 6, pp. 490-496, 2010.

[28] P. E. Polak, S. Kalinin, C. Dello Russo et al., "Protective effects of a peroxisome proliferator-activated receptor- $\beta / \delta$ agonist in experimental autoimmune encephalomyelitis," Journal of Neuroimmunology, vol. 168, no. 1-2, pp. 65-75, 2005.

[29] P. Delerive, K. de Bosscher, S. Besnard et al., "Peroxisome proliferator-activated receptor $\alpha$ negatively regulates the vascular inflammatory gene response by negative cross-talk with transcription factors NF- $\kappa \mathrm{B}$ and AP-1," Journal of Biological Chemistry, vol. 274, no. 45, pp. 32048-32054, 1999.

[30] J. D. Ji, H. J. Kim, Y. H. Rho et al., "Inhibition of IL-10induced STAT3 activation by 15 -deoxy- $\Delta 12,14$-prostaglandin J2," Rheumatology, vol. 44, no. 8, pp. 983-988, 2005.

[31] J. T. Huang, J. S. Welch, M. Ricote et al., "Interleukin-4dependent production of PPAR- $\gamma$ ligands in macrophages by 12/15-lipoxygenase," Nature, vol. 400, no. 6742, pp. 378-382, 1999.

[32] M. D. Carter, S. Reese Harry, and D. W. Wright, "Identification of hydroxyeicosatetraenoic acid components of schistosomal hemozoin," Biochemical and Biophysical Research Communications, vol. 363, no. 3, pp. 867-872, 2007.

[33] J. L. Reyes and L. I. Terrazas, "The divergent roles of alternatively activated macrophages in helminthic infections," Parasite Immunology, vol. 29, no. 12, pp. 609-619, 2007.

[34] T. Kreider, R. M. Anthony, J. F. Urban Jr., and W. C. Gause, "Alternatively activated macrophages in helminth infections," Current Opinion in Immunology, vol. 19, no. 4, pp. 448-453, 2007. 
[35] W. Noël, G. Raes, G. H. Ghassabeh, P. de Baetselier, and A. Beschin, "Alternatively activated macrophages during parasite infections," Trends in Parasitology, vol. 20, no. 3, pp. 126-133, 2004.

[36] P. Loke, A. S. MacDonald, and J. E. Allen, "Antigen-presenting cells recruited by Brugia malayi induce Th2 differentiation of naive CD4+ T cells," European Journal of Immunology, vol. 30, no. 4, pp. 1127-1135, 2000.

[37] P. N. Loke, A. S. MacDonald, A. Robb, R. M. Maizels, and J. E. Allen, "Alternatively activated macrophages induced by nematode infection inhibit proliferation via cell-to-cell contact," European Journal of Immunology, vol. 30, no. 9, pp. 2669-2678, 2000.

[38] R. M. Anthony, J. F. Urban, F. Alem et al., "Memory TH2 cells induce alternatively activated macrophages to mediate protection against nematode parasites," Nature Medicine, vol. 12, no. 8, pp. 955-960, 2006.

[39] N. Mejri and B. Gottstein, "Intraperitoneal Echinococcus multilocularis infection in C57BL/6 mice affects CD40 and B7 costimulator expression on peritoneal macrophages and impairs peritoneal T cell activation," Parasite Immunology, vol. 28, no. 8, pp. 373-385, 2006.

[40] L. I. Terrazas, D. Montero, C. A. Terrazas, J. L. Reyes, and M. Rodríguez-Sosa, "Role of the programmed Death-1 pathway in the suppressive activity of alternatively activated macrophages in experimental cysticercosis," International Journal for Parasitology, vol. 35, no. 13, pp. 1349-1358, 2005.

[41] P. Smith, C. M. Walsh, N. E. Mangan et al., "Schistosoma mansoni worms induce anergy of $\mathrm{T}$ cells via selective upregulation of programmed death ligand 1 on macrophages," Journal of Immunology, vol. 173, no. 2, pp. 1240-1248, 2004.

[42] S. L. Friedman, "Hepatic fibrosis-overview," Toxicology, vol. 254, no. 3, pp. 120-129, 2008.

[43] S. L. Friedman, "Liver fibrosis-from bench to bedside," Journal of Hepatology, vol. 38, supplement 1, pp. S38-S53, 2003.

[44] S. L. Friedman, "Molecular regulation of hepatic fibrosis, an integrated cellular response to tissue injury," Journal of Biological Chemistry, vol. 275, no. 4, pp. 2247-2250, 2000.

[45] H. She, S. Xiong, S. Hazra, and H. Tsukamoto, "Adipogenic transcriptional regulation of hepatic stellate cells," Journal of Biological Chemistry, vol. 280, no. 6, pp. 4959-4967, 2005.

[46] J. T. M. Tan, S. V. McLennan, W. W. Song et al., "Connective tissue growth factor inhibits adipocyte differentiation," American Journal of Physiology, vol. 295, no. 3, pp. C740-C751, 2008.

[47] S. Hazra, S. Xiong, J. Wang, R. A. Rippe, V. K. K. Chatterjee, and H. Tsukamoto, "Peroxisome proliferator-activated receptor $\gamma$ induces a phenotypic switch from activated to quiescent hepatic stellate cells," Journal of Biological Chemistry, vol. 279, no. 12, pp. 11392-11401, 2004.

[48] B. Anthony, J. T. Allen, Y. S. Li, and D. P. McManus, "Hepatic stellate cells and parasite-induced liver fibrosis," Parasites and Vectors, vol. 3, no. 1, article 60, 2010.

[49] P. B. Bartley, G. A. Ramm, M. K. Jones, R. G. Ruddell, Y. Li, and D. P. McManus, "A contributory role for activated hepatic stellate cells in the dynamics of Schistosoma japonicum egginduced fibrosis," International Journal for Parasitology, vol. 36, no. 9, pp. 993-1001, 2006.

[50] D. Chang, L. N. Z. Ramalho, F. S. Ramalho, A. L. C. Martinelli, and S. Zucoloto, "Hepatic stellate cells in human schistosomiasis mansoni: a comparative immunohistochemical study with liver cirrhosis," Acta Tropica, vol. 97, no. 3, pp. 318-323, 2006.
[51] H. Chen, Y. W. He, W. Q. Liu, and J. H. Zhang, "Rosiglitazone prevents murine hepatic fibrosis induced by Schistosoma japonicum," World Journal of Gastroenterology, vol. 14, no. 18, pp. 2905-2911, 2008.

[52] B. Anthony, W. Mathieson, W. de Castro-Borges, and J. Allen, "Schistosoma mansoni: egg-induced downregulation of hepatic stellate cell activation and fibrogenesis," Experimental Parasitology, vol. 124, no. 4, pp. 409-420, 2010.

[53] T. A. Wynn, "Fibrotic disease and the TH1/TH2 paradigm," Nature Reviews Immunology, vol. 4, no. 8, pp. 583-594, 2004. 


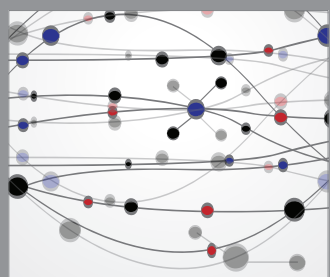

The Scientific World Journal




Gastroenterology

Research and Practice
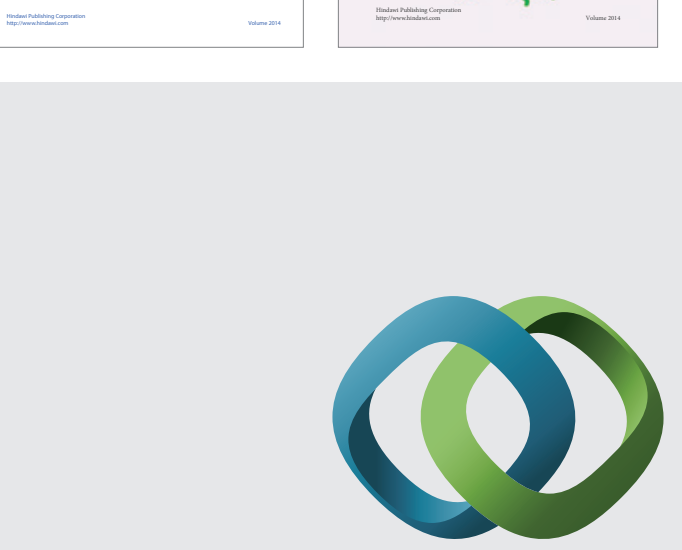

\section{Hindawi}

Submit your manuscripts at

http://www.hindawi.com


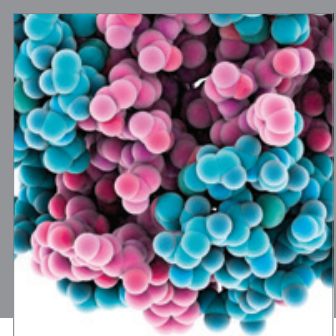

Journal of
Diabetes Research



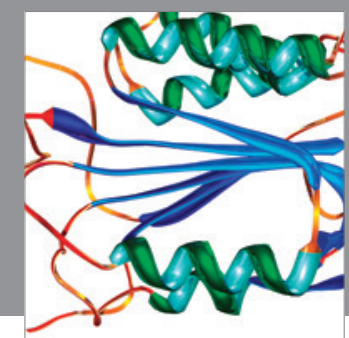

Disease Markers
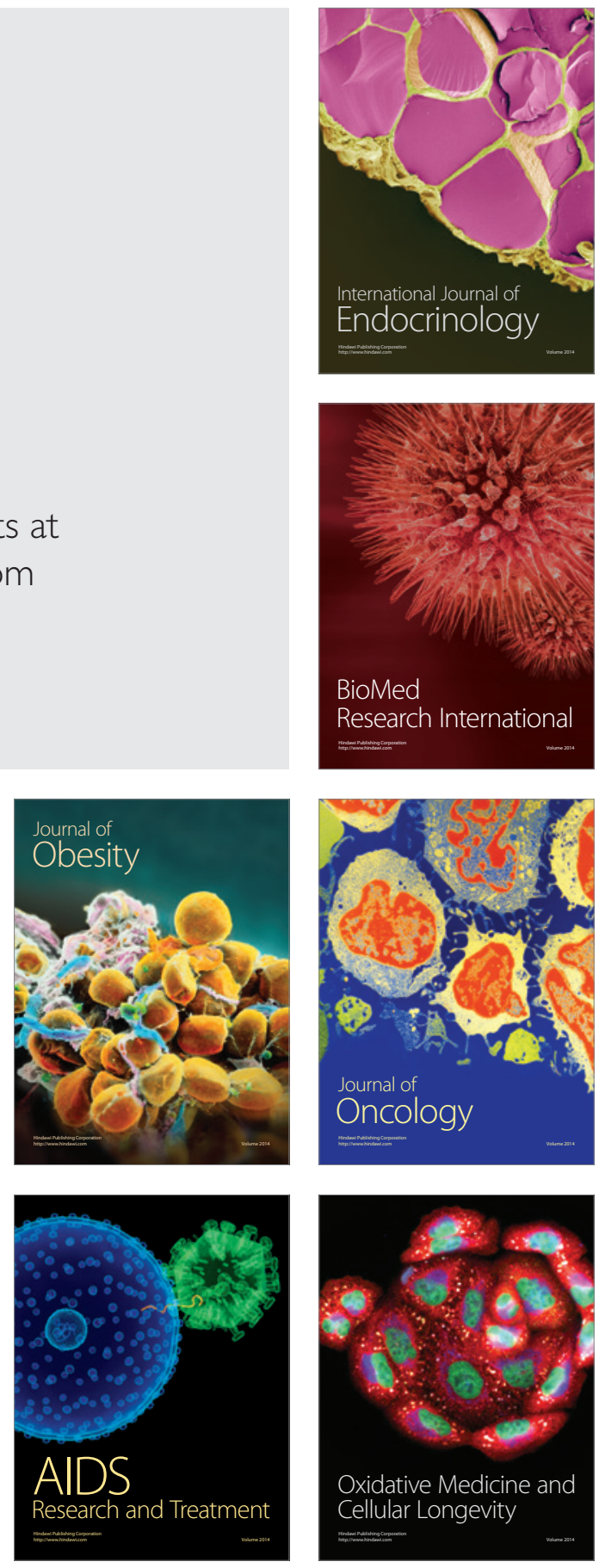